\title{
A Novel Time-Delay Estimation Methodology Based on Spread Spectrum Communications
}

\author{
Wen Zhun Huang ${ }^{\mathrm{a}}{ }^{*}$, Xin Xin Xie ${ }^{\mathrm{b}}$ and Shan Wen Zhang ${ }^{\mathrm{c}}$ \\ ${ }^{1}$ Department of Electronic Information Engineering, Xijing University, Xi'an 710123, China

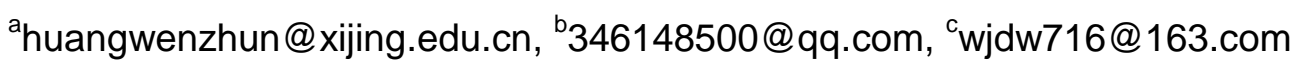

\begin{abstract}
Keywords: Channel Estimation Techniques, Spread Spectrum Communications, Least Squares Estimator, Time-Delay Estimation, underwater acoustic communication
\end{abstract}

\begin{abstract}
In this paper, we conduct research on effective and robust time-delay estimation methodology for spread spectrum communications. The development of underwater acoustic spread spectrum communication technology derived from the demand for underwater remote, reliable communication. Due to the underwater acoustic channel belt width, background noise and high rate of Doppler frequency shift, severe multipath effect, absorption problems such as uneven, makes the communication quality serious decline. We firstly analyze the fast time-delay estimators to serve as the prior knowledge, and later, we analyze the channel estimation techniques. Finally, we test the error and efficiency. Results show that the system has better ability to resist noise and multiple access interference, and improve the chaotic spread spectrum sequence to better the performance of the system of traditional chaotic spread spectrum sequence. The experimental result proves the effectiveness of proposed methodology. In the future, we plan to conduct more literature reviews to modify the current technique.
\end{abstract}

\section{Introduction}

Spread spectrum communication technology widely used in communications and navigation. With the increasingly development of science and technology, people's increasing demand to communication, especially the pursuit of stability to the rapid development of the spread spectrum communication technology [1]. The development of underwater acoustic spread spectrum communication technology derived from the demand for underwater remote, reliable communication. Due to the underwater acoustic channel belt width, background noise and high rate of doppler frequency shift, severe multipath effect, absorption problems such as uneven, makes the communication quality serious decline. The spread spectrum communication technology is introduced into the underwater acoustic communication, therefore, become the effective way to solve the stability of the communication. Spread spectrum communication technology itself is not sensitive to frequency offset, but because of the underwater acoustic signal transmission rate is low, so relative to the wireless communication, electromagnetic wave doppler frequency shift of underwater acoustic communication rate is very high [2-5]. Underwater acoustic communication are commonly use search method, namely after capture using the local carrier wave with different frequency to signal demodulation, choose the best carrier in order to realize the carrier synchronization, but the complexity of the algorithm is higher, the general real-time system can use this method. So the doppler frequency shift are estimated directly became a kind of effective method of underwater acoustic spread spectrum communication system, based on chirp Z transform (CZT) algorithm for doppler frequency shift of underwater acoustic spread spectrum communication system estimate method, for the processing of doppler frequency shift only considered the effect of frequency shift, and for the expansion of the doppler shift effect didn't make a corresponding analysis and consideration [6-9].

In this paper, we hope that through the super - coherent estimates for better performance than the traditional method, because we use voice task: at the same time, looking for the biggest point of 
ambiguity function of symmetrical. Here, we introduce some links on cross-correlation function and investigation show that the first derivative test performs well rather than a more optimal numerical process a maximum of search function. We consider a satellite communication scenarios DS/SS CDMA system, especially our reference (the next) European Galileo communication scenarios. Galileo will use a binary offset carrier the bank of China $(m, n)$ modulation, in order to avoid interference with the existing GPS system, reduce the multipath effect and precision to achieve synchronization by offering this narrow peak modulation $[10,11]$.

\section{The Proposed Methodology}

The Fast Time-Delay Estimators. Time delay estimation problem is to determine the purpose of between two scaled versions of the same signal delay. It is proposed in this paper an adaptive doppler estimation and compensation algorithm, launch a training signal, this section of the signal sending and receiving ends are known, carrier synchronization, first of all, the local training signal resampling, then with the receiving signal multiplication algorithm. Through the analysis of the algorithm to signal adjustment coefficient of resampling until the local training signal and received signal does not exist the doppler frequency shift, end complete carrier synchronization process system. This involves sampling and processing two continuous time signals denoted in the Eq.1.

$$
\begin{aligned}
& x_{1}(t)=s(t)+n_{1}(t) \\
& x_{2}(t)=A \cdot s(t-D)+n_{2}(t)
\end{aligned}
$$

From the spectrum utilization point of view, means to reduce bandwidth with reference to the complexity of equipment cost, in order to get the same total system gain; Consider to reduce the volume, weight and the launch and the receiving end to improve spectrum efficiency, stability and reliability of the system, choose the time-division duplex working mode; In order to guarantee a certain error resistance, and use the field programmable gate display to complete signal demodulation, using four phase shift keying modulation, non-coherent demodulation method. Spread spectrum (spread spectrum, SS) system is to use a high speed, large bandwidth digital coding of pseudo random sequence to directly modulated carrier signal, so that the rf signal spectrum width. In direct sequence spread spectrum (direct sequence spread spectrum, DSSS) CDMA (code division multiple access, CDMA) system, one of the most key technology is the choice of spread spectrum sequence and structure, the length of the element, the relevant features, randomness decides the performance of the system, such as strong anti-interference performance of the system, resistance to multipath effect of fading resistance, low interception rate, high precision timing, data hiding and confidential information and is closely related to the adopted by the spread spectrum sequence. Therefore, let us now define the estimated cross-correlation function between the two signals in the Eq.2.

$$
R(\tau)=\frac{1}{N} \sum_{k=1}^{N} x_{1}\left(k T_{c}\right) \cdot x_{2}\left(k T_{c+\tau}\right)
$$

If Nyquist conditions apply, resolution is usually more sampling period and some form of interpolation. Commonly used technology, the original Jacovitti Scarano and proposed. The parabola fitted over these three samples as Eq.3.

$$
D_{p}=-\frac{\theta}{2} \cdot \frac{R_{1}-R_{-1}}{R_{1}-2 \cdot R_{0}+R_{-1}}
$$

Unfortunately, in low signal-to-noise ratio is not the case cause serious performance degradation. Therefore, in this case, it may be useful for time delay estimation using another method introduced in the literature. In particular, because fuzzy function is symmetrical, the biggest around the consistent 
estimator of the median can also detect their biggest. As is known to all, in fact, the biggest delay estimation using the Hilbert transform find task is converted to a find a zero crossing. The accuracy of the voice in spread spectrum communication is a key problem is satisfied, because it is often with many of the digital communication system performance, in the case of satellite positioning, the user's location accuracy is directly related to the accuracy of the voice. In satellite communications, such as in the GPS and Galileo, DS/SS in the physical modulation communication. Adjustment in the process of acquisition of synchronization in the chip, or less, and the tracking process in sync. Synchronization related, therefore, the task of the receiver including initial (starting data recovery session) on their despreading reference and spread to reach and maintain the code signal, and the precise synchronization between them in the subsequent data receiving time. The general corresponding structure could be seen in the Fig. 1.

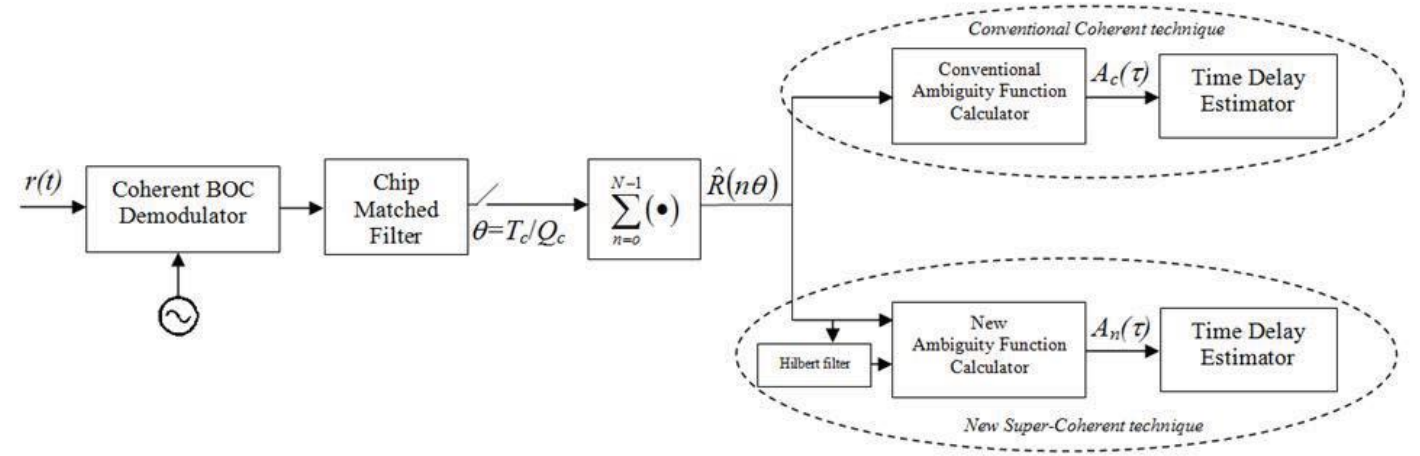

Fig. 1 The Flowchart of the Prior Method

Here, since we are dealing with BOC pulses, we double the chip rate in respect to the GPS systems, meaning that we consider two samples per pulse, i.e. four samples per chip. This technique has the advantage that the software is convenient to transplant, short development cycle, can be in the same device to realize a variety of purposes.

The Doppler estimation. According to section 2 of the doppler analysis and modeling of underwater acoustic channel, this paper proposes a doppler estimation and compensation algorithm. In this paper, the doppler signal estimation method for launch a training, including training sequence is fixed and in sending and receiving ends are known, and for the sake of simplicity, here the training sequence is assumed to be 1 . System through a preliminary estimates the doppler frequency shift rate, then the local training according to the signal resampling rate of doppler frequency shift. After the signal capture, the training of the local signal and received signal multiplication algorithm to complete this training signal process. Signal after the expansion of this solution contains doppler shift and estimates the doppler frequency shift of the difference between the frequency of information. This signal analysis of the spectrum, if given the results of the corresponding with the zero frequency deviation is modified to estimate doppler offset value, repeat the process until the estimated results with real results in a permitted range. As you can see, actually the baseband signal containing the doppler frequency transposition, but the signal-to-noise ratio is very low, will not be able to directly extract frequency deviation information, we can take advantage of the spread spectrum system has the nature of the spread spectrum gain algorithm to deal with the signals are analyzed in order to improve the signal-to-noise ratio.

For spectrum analysis, the most commonly used method is the Fourier transform, signal into frequency domain to time domain. For digital signal is discrete Fourier transform (DFT). Selection of estimate is an adaptive process denoted as the local doppler frequency shift estimation from the initial value began to adjust. The changeable parameters are denoted as Eq.4. 


$$
\Delta_{n}=\left\{\begin{array}{cc}
\Delta_{n-1}-\mu \Phi(\eta), & n \geq 2 \\
\Delta_{0}, & n=1
\end{array}\right.
$$

Only to compensate the signal in frequency is not enough, the doppler compensation method must be able to offset the expansion of the doppler effect. General in the system is achieved by adjusting the system sampling rate and the received signal synchronization, but in a communication system based on software radio, due to the limitations of the hardware design, the resolution of the local sampling rate not so high, could not reach the requirements of adjustment.

The proposed methodology. Referring to the same system model of the previous Section, we now select 5 samples of the cross-correlation function to use in our procedure. Scheme of the interpolation functions is shown in the Fig. 2.
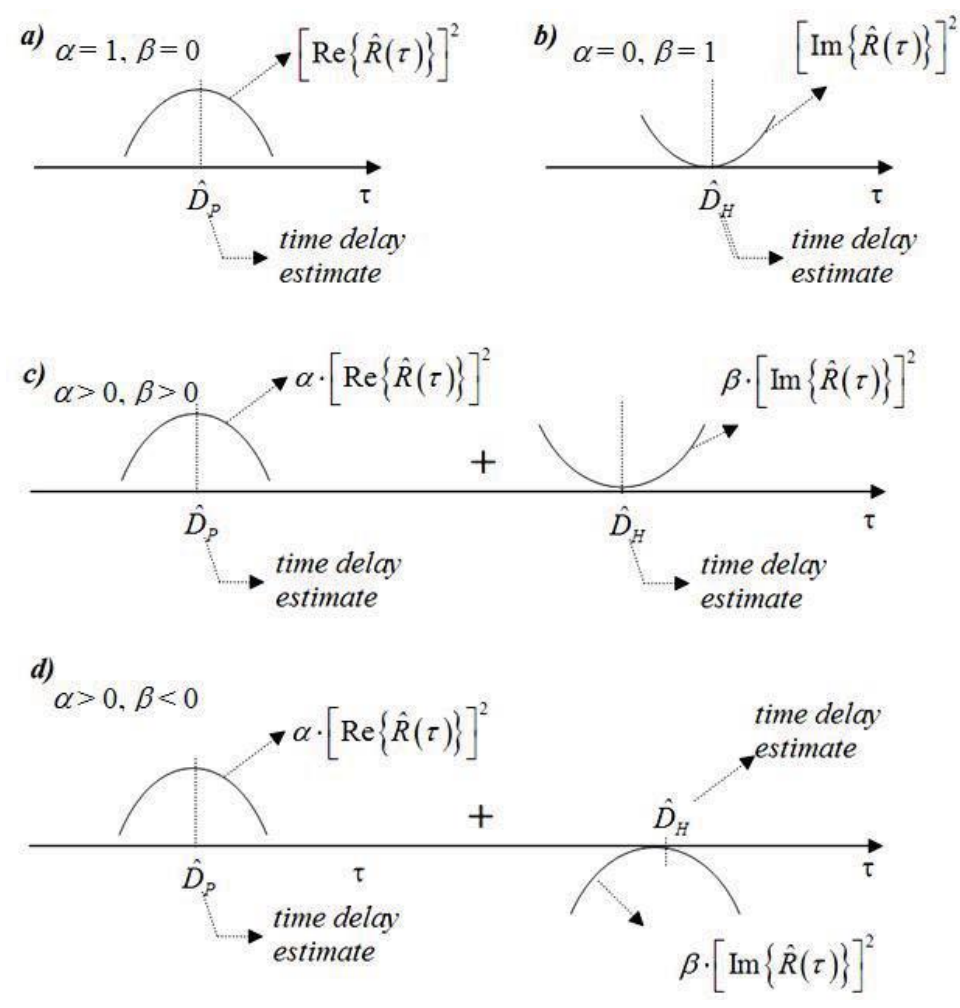

Fig. 2 The scheme of the interpolation functions

Following the same notation of the previous Section, we denote as Eq.5.

$$
R_{-2}=R\left(\tau_{m}-2 \theta\right), R_{2}=R\left(\tau_{m}+2 \theta\right)
$$

Five samples of cross-correlation of the minimum number of samples we can take advantage of the real and imaginary part of complex envelope cross-correlation function of the voice. In fact, we now can be inserted into two parabolic functions (a real and imaginary part of the cross-correlation). Then, we gained the linear combination of these two parabolic new ambiguity functions, therefore the new alarm. First parabola function, we use the same method of traditional coherent method but now parabolic fitting in one of three samples. The second parabola function is used for all five sample. RAKE receiver for indoor transmission signal, when the propagation delay of more than a yard piece, the length of the multipath signals can be regarded as is related, which can synthesize different path to the output of the receiver a output, in order to provide better than single-channel correlator signal detection, and then on the basis of this over to reconcile the sentence. But generally the RAKE receiver is implemented on baseband, did not consider multipath signals received by the carrier phase, between therefore will not be able to eliminate the influence of the phase difference, which affects positioning 
accuracy. In general, there are two factors affecting the performance of RAKE receiver: multipath component selection mode, the multipath merger way. The author based on the modified Walsh code puts forward a kind of improved type RAKE reception scheme, and using Matlab/Simulink software to verify the system BER performance under Rayleigh fading channel. It must be pointed out that this second parabola should be implemented in an unlimited number of samples, because the Hilbert filter is a kind of IIR filter. Underwater acoustic spread spectrum communication system based on software radio system has the following characteristics: first, using the general hardware structure, in addition to transducer and several/a conversion module and the system of the frequency band corresponding to the outside, other parts are of universal design. Second, all modules are implemented by software system, modularization, easy to transplant, short development cycle, can adjust the system parameters according to the actual situation.

The output of the filtering process is the Hilbert transform of the crosscorrelation function. We can obtain the second parabola squaring the equation of the line fitted on these two samples. This is the reason why we need 5 samples of the cross-correlation function otherwise, during the filtering process, fewer samples would not benefit of the full length of the Hilbert FIR filter.

In order to satisfy the high efficiency of modern communication system for communication, and the requirement of security in this paper, a parallel combination of spread spectrum communication system based on pulse modulation, pulse modulation UWB communication and communication respectively in secrecy performance of parallel combination expansion and the advantages of efficient communication ability, make the new system can also have effective communication ability and good security performance. Design, the system model is established, and by using the simulation software simulation and analysis on the performance of the system. System in order to prevent an infinite loop, set a threshold, when the cycles to reach the threshold value, system exit estimation process, but pay attention to the estimate of result is not necessarily correct at this time. In the next section, we will discuss the experiment.

\section{Experiment and Simulation Result}

In this section, the performance of the proposed methodology is evaluated. A few test validated the theory expression and statistical model of assumptions (in the previous section) asymptotic properties of the interpolation method is proposed. Simulation scenario to consider our work refers to satellite DS/SS CDMA communication, especially in communication (the next) European Galileo scenario.

In spread spectrum sequence cycle is constant, the $\mathrm{K}$ value, the greater the system of information transmission rate is higher, but the system performance decreases, conform to the contradictory relationship between validity and reliability of communication system. So, in the case that could satisfy the requirement of a certain level of bit error rate, can change the $\mathrm{M} / \mathrm{r}$ value or change at the same time of $\mathrm{M}$ and $\mathrm{r}$ values for different information transmission rate and realize the effective transmission of information. At the same time, the system USES the narrow pulse transmission information, and can obtain a lower detection and intercept probability and improve the security performance of the system. Fig. 3 shows the experimental sets one. 


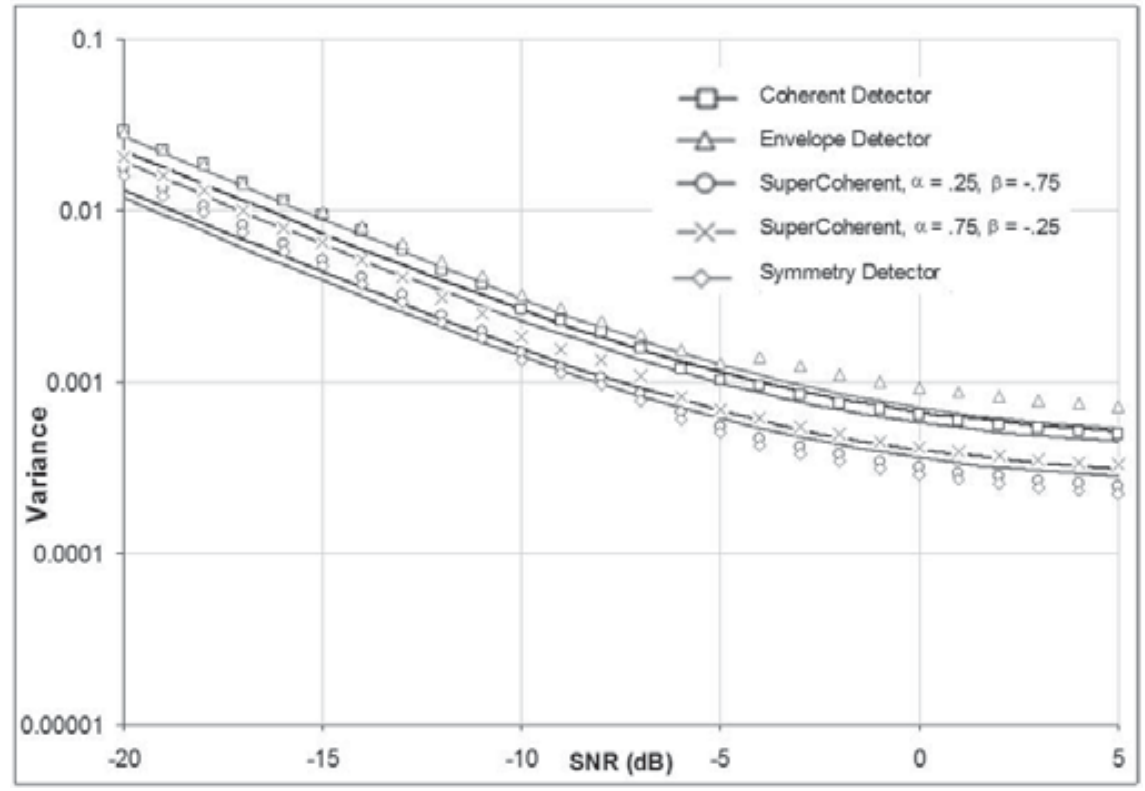

Fig. 3 The Experiment Result

\section{Summary}

In this paper, starting from the analysis of underwater acoustic channel, the doppler frequency shift and its influence on the spread spectrum communication system modeling, and fully considering the doppler frequency shift in the underwater acoustic channel. And then puts forward an adaptive doppler estimation and compensation algorithm, the algorithm can meet the real-time communication conditions accurately to offset the effect of doppler frequency shift. And the sequence was applied to multi-user chaotic spread spectrum communication system, and gives the improved chaotic spread spectrum sequence and multi-user chaotic spread spectrum communication system simulation model, and the guide of theories of BER. Then the multi-user chaotic spread spectrum communication system has carried on the detailed performance analysis, including the system bit error rate with the change of signal to noise ratio, improve the fractal parameters of chaotic spread spectrum sequence and the influence of the initial value system bit error rate, as well as the system bit error rate with the change of the number of users.

\section{Acknowledgements}

This work is financially supported by the scientific research program of Shaanxi Provincial Education Department (Program No. 14JK2156).

\section{References}

[1] J. H. Du, C. W. Yuan and J. B. Zhang: IET Communications, Vol. 8 (2014) No. 12, p.2193.

[2] Christensen, Eric, and M. Hanson: U.S. Patent 8,655,086. (2014).

[3] Domingues, Patricio: International Conference on Computer Science and Applications (Sanya, Hainan, China, 2014), Vol. 52 (2014), p.560.

[4] L. Dai, Y. S. Zhang, Y. J. Li and H. X. Wang: Sixth International Conference on Digital Image Processing (Athens, 2014), p.915906.1.

[5] J. H. Park, H. C. Byeong and J. W. Kim: U.S. Patent 8,699,804. (2014).

[6] A. K. Bhandari, A. Kumar and G. K. Singh: Expert Systems with Applications, Vol. 42 (2015) No.3, p.1573. 
[7] G. K. Kanungo, N. Singh, J. Dash and A. Mishra: Intelligent Computing, Communication and Devices (Springer India, India, 2015), P.403.

[8] A. Singla, and S. Patra: Computational Intelligence in Data Mining-Volume 2 (Springer India, India 2015), P.19.

[9] D. Mandal, A. Chatterjee and M. Maitra: Advanced Computing, Networking and Informatics Volume 1 (Springer International Publishing, Switzerland 2014), p.267.

[10] Aziz, N. Abdullah: Journal of Engineering and Technological Sciences, Vol. 46 (2014) No.1, p. 102.

[11] Leder, Kevin: Cell, Vol. 156 (2014) No. 3, p. 603. 